The generalized Jacobi equation

This article has been downloaded from IOPscience. Please scroll down to see the full text article.

2002 Class. Quantum Grav. 194231

(http://iopscience.iop.org/0264-9381/19/16/301)

View the table of contents for this issue, or go to the journal homepage for more

Download details:

IP Address: 128.206.162.204

The article was downloaded on 23/09/2010 at 19:19

Please note that terms and conditions apply. 


\title{
The generalized Jacobi equation
}

\author{
C Chicone $^{1}$ and B Mashhoon ${ }^{2}$ \\ ${ }^{1}$ Department of Mathematics, University of Missouri-Columbia, Columbia, MO 65211, USA \\ 2 Department of Physics and Astronomy, University of Missouri-Columbia, Columbia, \\ MO 65211, USA \\ E-mail: mashhoonb@missouri.edu
}

Received 27 March 2002

Published 30 July 2002

Online at stacks.iop.org/CQG/19/4231

\begin{abstract}
The Jacobi equation in pseudo-Riemannian geometry determines the linearized geodesic flow. The linearization ignores the relative velocity of the geodesics. The generalized Jacobi equation takes the relative velocity into account; that is, when the geodesics are neighbouring but their relative velocity is arbitrary the corresponding geodesic deviation equation is the generalized Jacobi equation. The Hamiltonian structure of this nonlinear equation is analysed in this paper. The tidal accelerations for test particles in the field of a plane gravitational wave and the exterior field of a rotating mass are investigated. In the latter case, the existence of an attractor of uniform relative radial motion with speed $2^{-1 / 2} c \approx 0.7 c$ is pointed out. The astrophysical implication of this result for the terminal speed of a relativistic jet is briefly explored.
\end{abstract}

PACS numbers: $0420 \mathrm{C}, 9858 \mathrm{~F}$

\section{Introduction}

The analysis of observations in Einstein's general relativity theory is a rather complicated issue in contrast with the elegant simplicity of the geometric structure of the theory [1]. Take, for instance, the excess perihelion precession of Mercury that was explained by Einstein in 1915 and provided the first major success of general relativity. This standard theoretical result follows from the solution of the geodesic equation in the post-Newtonian approximation. However, the relevant observational results are usually obtained by monitoring the motion of Mercury from the Earth via the electromagnetic radiation that is reflected by Mercury and reaches astronomical telescopes on the Earth. In effect, one has to deal with the relative motion of one geodesic ('Mercury') with respect to the other ('Earth') [2]. Ideally, the observer following the reference geodesic sets up in its neighbourhood a laboratory, where the measuring devices are assumed to work as in inertial spacetime. This local inertial frame of the observer is represented by the Fermi coordinate system. To construct such a coordinate 
patch, an orthonormal set of ideal gyroscopes would be required to define the local spatial frame of the observer, while an ideal standard clock would measure the observer's proper time. The reduced equation of geodesic motion of a nearby particle in such a system is the generalized Jacobi equation that will be studied in this paper. The results may be applied to high-energy astrophysical phenomena as well as precision measurements in space involving the tidal field of the Earth or an incident gravitational wave.

In a congruence of timelike geodesics, neighbouring curves have rates of separation that are usually negligible compared to the speed of light in vacuum $c$. In this case, the geodesic deviation equation is the standard Jacobi equation [3]. There are physical circumstances, however, where the worldlines of adjacent geodesics diverge rapidly. Consider, for instance, a congruence of test particles with a common initial velocity falling towards a black hole such that, depending on their impact parameters, some particles would be captured by the black hole, while others would escape its gravitational pull. Though the geodesics are neighbouring as they approach the black hole, the rate of separation between the particles that fall in and those that are merely deflected would no longer be small compared to $c$. This example makes it clear that the relevant equation of relative motion, i.e., the generalized Jacobi equation, has a limited domain of applicability, since rapidly diverging geodesics would no longer be adjacent after a period of time that is characteristic of the spacetime curvature-that is, $\varrho / c$, where $\varrho$ is the radius of curvature.

The investigation of the relative motion of neighbouring observers following arbitrary timelike curves leads to the general deviation equation. The relativistic theory of tides is based on the general geodesic deviation equation. This is rather complicated when the rate of separation of geodesics could be close to the speed of light; therefore, we confine our detailed discussion here to a limited form of this equation that is valid to first order in the relative separation (i.e., the generalized Jacobi equation). On the other hand, in the ('nonrelativistic') case where a smooth geodesic congruence is given, a general treatment of the geodesic deviation equation is available; in fact, a new derivation can be found in the recent work of Kerner et al [4] who also give a novel application: they use the geodesic deviation equation together with Poincaré's perturbation method to approximate bounded (eccentric) geodesics in the Schwarzschild metric that are close to circular geodesics.

The generalized Jacobi equation is discussed in detail in section 2; in particular, we are interested in the dynamical system represented by this nonlinear equation, where the nonlinearity is due to the existence of velocity-dependent terms in the system. Therefore, we present a detailed analysis of its Hamiltonian structure.

To illustrate certain general dynamical features of the generalized Jacobi equation, we consider the relative motion of neighbouring test particles in the field of a plane gravitational wave in section 3 and in the exterior field of a gravitating source in section 4 . We note that many compact astrophysical systems emit powerful oppositely-directed jets that are highly collimated. No basic theory of astrophysical jets is available at present, though manyespecially magnetohydrodynamic_-models have been proposed. In particular, the physical processes that determine the asymptotic speed of the bulk flow are not clear at this time. Of particular interest in this connection is the relevance of the relative radial motion described in section 4 to the 'terminal' bulk motion of a relativistic jet once such an outflow is beyond the active region around the source and the bulk motion is subject only to the gravitational attraction of the source. Taking only the relativistic tidal forces into account, we demonstrate in this case the presence of an 'attractor' at a Lorentz factor $\Gamma=\sqrt{2}$, corresponding to $V=c / \sqrt{2} \approx 0.7 c$, for the terminal bulk motion of a jet relative to the ambient medium.

Finally, section 5 contains a discussion of our results. In the following sections, we use units such that $c=1$. 


\section{Generalized Jacobi equation}

The generalized Jacobi equation in Fermi coordinates can be obtained simply by writing an alternative form of the geodesic equation, i.e., the reduced geodesic equation (2.5), in Fermi coordinates. However, to clarify the Hamiltonian and Lagrangian aspects of this equation, it is useful to proceed as in the following subsections.

\subsection{Hamiltonian and Lagrangian formalisms}

As is well known, the geodesic equation in local coordinates $x^{\mu}$ on a pseudo-Riemannian manifold with metric

$$
\mathrm{d} s^{2}=g_{\mu \nu}(x) \mathrm{d} x^{\mu} \mathrm{d} x^{\nu}
$$

is equivalent to the Euler-Lagrange equation for the Lagrangian

$$
\mathcal{L}\left(x^{\mu}, u^{\nu}\right)=\frac{1}{2} g_{\mu \nu}(x) u^{\mu} u^{\nu},
$$

where

$$
u^{\mu}:=\frac{\mathrm{d} x^{\mu}}{\mathrm{d} \lambda}
$$

the canonical momentum is given by

$$
p_{\mu}=\frac{\partial \mathcal{L}}{\partial u^{\mu}}=g_{\mu \nu}(x) u^{\nu},
$$

and $\lambda$ is an arbitrary parameter (see [5]). In fact, the Euler-Lagrange equation for this system is equivalent to

$$
\frac{\mathrm{d}^{2} x^{\mu}}{\mathrm{d} \lambda^{2}}+\Gamma_{\alpha \beta}^{\mu} \frac{\mathrm{d} x^{\alpha}}{\mathrm{d} \lambda} \frac{\mathrm{d} x^{\beta}}{\mathrm{d} \lambda}=0
$$

where

$$
\Gamma_{\alpha \beta}^{\mu}=\frac{1}{2} g^{\mu \gamma}\left(g_{\gamma \alpha, \beta}+g_{\gamma \beta, \alpha}-g_{\alpha \beta, \gamma}\right)
$$

are the Christoffel symbols. The corresponding Hamiltonian is

$$
\mathcal{H}\left(x^{\mu}, p_{\nu}\right)=p_{\mu} u^{\mu}-\mathcal{L}\left(x^{\mu}, u^{\nu}\right)=\frac{1}{2} g^{\mu \nu}(x) p_{\mu} p_{\nu},
$$

which is independent of the parameter $\lambda$; thus, $\mathcal{H}$ is constant on geodesics. It follows that along a geodesic $\lambda$ is a linear function of $s$.

\subsection{Isoenergetic reduction}

Let us specialize to a four-dimensional Lorentzian manifold where we take an admissible coordinate chart and the sign convention $(-,+,+,+)$ for the Lorentzian metric. We will also use the Einstein summation rules; Greek indices run from 0 to 3 and Latin indices run from 1 to 3. Moreover, round brackets around indices denote symmetrization, while square brackets denote antisymmetrization.

The timelike geodesics $\left((\mathrm{d} s / \mathrm{d} \lambda)^{2}<0\right)$ with energy $\mathcal{H}=-1 / 2$ have $\lambda=\tau$, where $\tau$ is the proper time along the geodesic such that $(\mathrm{d} s / \mathrm{d} \tau)^{2}=-1$. We will determine the isoenergetic reduction for the Hamiltonian system restricted to this manifold.

The Hamiltonian equations of motion (where, for simplicity, we have assumed that $p^{\mu}=u^{\mu}$; that is, the mass of the free test particle is normalized to unity) are given by

$$
\begin{aligned}
\frac{\mathrm{d} x^{\mu}}{\mathrm{d} \tau} & =\frac{\partial \mathcal{H}}{\partial p_{\mu}}=g^{\mu \nu} p_{v}=p^{\mu}, \\
\frac{\mathrm{d} p_{\mu}}{\mathrm{d} \tau} & =-\frac{\partial \mathcal{H}}{\partial x^{\mu}}=-\frac{1}{2} g^{\alpha \beta}{ }_{, \mu} p_{\alpha} p_{\beta} .
\end{aligned}
$$


Reduction is accomplished by first separating the pairs of canonically conjugate variables $\left(x^{\mu}, p_{v}\right)$ into time and space variables; that is, we take $\left(x^{\mu}, p_{v}\right)=\left(t, p_{0} ; x^{i}, p_{j}\right)$ so that points in spacetime with energy $-1 / 2$ satisfy the equation

$$
\mathcal{H}\left(t, p_{0} ; x^{i}, p_{j}\right)=\frac{1}{2} g^{00} p_{0}^{2}+g^{0 i} p_{0} p_{i}+\frac{1}{2} g^{i j} p_{i} p_{j}=-\frac{1}{2} .
$$

Because the flow of time does not stop along the worldline of an observer, we have $\mathrm{d} t / \mathrm{d} \tau \neq 0$. Hence, by Hamilton's equations, the partial derivative $\partial \mathcal{H} / \partial p_{0}$ does not vanish. By the implicit function theorem, there is a function $\alpha$ such that

$$
\mathcal{H}\left(t, \alpha\left(t, x^{i}, p_{j}\right), x^{i}, p_{j}\right)=-\frac{1}{2} .
$$

That is, the energy surface is (locally) the graph of $\alpha$. Of course, we can also solve explicitly for $\alpha$. Indeed, because $g^{00} \neq 0$ in an admissible chart we can complete the square with respect to $p_{0}$ in equation (2.2) to obtain

$$
\left(p_{0}+\frac{g^{0 i} p_{i}}{g^{00}}\right)^{2}=-\frac{1+\tilde{g}^{i j} p_{i} p_{j}}{g^{00}},
$$

where

$$
\tilde{g}^{i j}:=g^{i j}-\frac{g^{0 i} g^{0 j}}{g^{00}}
$$

Hence,

$$
\alpha\left(t, x^{i}, p_{j}\right)=-\frac{g^{0 i}}{g^{00}} p_{i} \pm\left(\frac{1+\tilde{g}^{i j} p_{i} p_{j}}{\left(-g^{00}\right)}\right)^{1 / 2} .
$$

We note that $\left(\tilde{g}^{i j}\right)$ is the inverse of the spatial metric $\left(g_{i j}\right)$; that is, $\tilde{g}^{i k} g_{k j}=\delta_{j}^{i}$.

On the energy surface, the equation

$$
\frac{\mathrm{d} p_{0}}{\mathrm{~d} \tau}=-\frac{\partial \mathcal{H}}{\partial t}\left(t, \alpha\left(t, x^{i}, p_{j}\right), x^{i}, p_{j}\right)
$$

decouples from the system (2.1). By computing the partial derivatives with respect to $x^{i}$ and $p_{i}$ on both sides of equation (2.3), we obtain the identities

$$
\frac{\partial \mathcal{H}}{\partial p_{0}} \frac{\partial \alpha}{\partial x^{i}}+\frac{\partial \mathcal{H}}{\partial x^{i}}=0, \quad \frac{\partial \mathcal{H}}{\partial p_{0}} \frac{\partial \alpha}{\partial p_{i}}+\frac{\partial \mathcal{H}}{\partial p_{i}}=0 .
$$

Using these relations, the Hamiltonian system (2.1) for the spatial variables can be expressed as

$$
\frac{\mathrm{d} x^{i}}{\mathrm{~d} \tau}=\frac{\partial \mathcal{H}}{\partial p_{i}}=-\frac{\partial \mathcal{H}}{\partial p_{0}} \frac{\partial \alpha}{\partial p_{i}}, \quad \frac{\mathrm{d} p_{i}}{\mathrm{~d} \tau}=-\frac{\partial \mathcal{H}}{\partial x^{i}}=\frac{\partial \mathcal{H}}{\partial p_{0}} \frac{\partial \alpha}{\partial x^{i}},
$$

where $\partial \mathcal{H} / \partial p_{0}=\mathrm{d} t / \mathrm{d} \tau \neq 0$ since $t$ is the timelike variable. Hence, the remaining 'spatial' equations in the Hamiltonian system (2.1) can be recast into the time-dependent Hamiltonian system ('the isoenergetic reduction')

$$
\frac{\mathrm{d} x^{i}}{\mathrm{~d} t}=-\frac{\partial \alpha}{\partial p_{i}}\left(t, x^{j}, p_{k}\right), \quad \frac{\mathrm{d} p_{i}}{\mathrm{~d} t}=\frac{\partial \alpha}{\partial x^{i}}\left(t, x^{j}, p_{k}\right),
$$

with Hamiltonian $-\alpha$.

The Hamiltonian isoenergetic reduction procedure is equivalent to a Lagrangian procedure. In fact, let us define the Lagrangian [2]

$$
L\left(t, x^{i}, v^{j}\right)=-\left[-\left(g_{00}(t, x)+2 g_{0 i}(t, x) v^{i}+g_{i j}(t, x) v^{i} v^{j}\right)\right]^{1 / 2},
$$


where $v^{i}:=\mathrm{d} x^{i} / \mathrm{d} t$, the minus sign under the square root ensures that $L$ is real and the other minus sign is to conform with the sign convention used in the Hamiltonian system (2.4). We now have

$$
p_{i}:=\frac{\partial L}{\partial v^{i}}=\left(g_{0 i}+g_{i j} v^{j}\right)\left[-\left(g_{00}+2 g_{0 i} v^{i}+g_{i j} v^{i} v^{j}\right)\right]^{-1 / 2}
$$

and the corresponding time-dependent Hamiltonian

$$
H=H\left(t, x^{i}, p_{j}\right):=p_{i} v^{i}-L\left(t, x^{i}, v^{j}\right)
$$

is given by

$$
\begin{aligned}
H & =-\left(g_{00}+g_{0 i} \frac{\mathrm{d} x^{i}}{\mathrm{~d} t}\right) \frac{\mathrm{d} t}{\mathrm{~d} \tau} \\
& =-\left(g_{00} p^{0}+g_{0 i} p^{i}\right)=-g_{0 \alpha} p^{\alpha}=-p_{0},
\end{aligned}
$$

where we have used the fact that the proper time $\tau$ along the geodesic worldline is such that $L=-\mathrm{d} \tau / \mathrm{d} t$. Hence $H=-\alpha$ in system (2.4).

This reduced system is equivalent to the second-order differential equation

$$
\frac{\mathrm{d}^{2} x^{i}}{\mathrm{~d} t^{2}}-\left(\Gamma_{\alpha \beta}^{0} \frac{\mathrm{d} x^{\alpha}}{\mathrm{d} t} \frac{\mathrm{d} x^{\beta}}{\mathrm{d} t}\right) \frac{\mathrm{d} x^{i}}{\mathrm{~d} t}+\Gamma_{\alpha \beta}^{i} \frac{\mathrm{d} x^{\alpha}}{\mathrm{d} t} \frac{\mathrm{d} x^{\beta}}{\mathrm{d} t}=0,
$$

which is the reduced geodesic equation. To prove this fact, note that the Hamiltonian or Lagrangian equations of motion are equivalent to the Euler-Lagrange equation

$$
\begin{aligned}
& \frac{\mathrm{d}}{\mathrm{d} t}\left[\left(g_{0 i}+g_{i j} v^{j}\right)\left[-\left(g_{00}+2 g_{0 i} v^{i}+g_{i j} v^{i} v^{j}\right)\right]^{-1 / 2}\right] \\
& =\frac{1}{2}\left(g_{00, i}+2 g_{0 j, i} v^{j}+g_{k l, i} v^{k} v^{\ell}\right)\left[-\left(g_{00}+2 g_{0 i} v^{i}+g_{i j} v^{i} v^{j}\right)\right]^{-1 / 2} .
\end{aligned}
$$

Let us use the proper time $\tau$ to recast equation (2.6) in the form

$$
\frac{\mathrm{d}}{\mathrm{d} \tau}\left(g_{i \alpha} u^{\alpha}\right)=\frac{1}{2} g_{\alpha \beta, i} u^{\alpha} u^{\beta},
$$

where $u^{\mu}=\mathrm{d} x^{\mu} / \mathrm{d} \tau$. After computation of the derivative by the product rule, the equation can be rearranged to the equivalent form

$$
g_{i \alpha} \frac{\mathrm{d} u^{\alpha}}{\mathrm{d} \tau}+\frac{1}{2}\left(g_{i \alpha, \beta}+g_{i \beta, \alpha}-g_{\alpha \beta, i}\right) u^{\alpha} u^{\beta}=0 .
$$

We multiply both sides of the last equation by $g^{\mu i}$ and use the identity $g^{\mu i} g_{i \alpha}+g^{\mu 0} g_{0 \alpha}=\delta_{\alpha}^{\mu}$ to show that

$$
\left(\delta_{\alpha}^{\mu}-g^{\mu 0} g_{0 \alpha}\right) \frac{\mathrm{d} u^{\alpha}}{\mathrm{d} \tau}+\frac{1}{2} g^{\mu i}\left(g_{i \alpha, \beta}+g_{i \beta, \alpha}-g_{\alpha \beta, i}\right) u^{\alpha} u^{\beta}=0 .
$$

Also, using the identity

$$
\Gamma_{\alpha \beta}^{\mu}=\frac{1}{2} g^{\mu i}\left(g_{i \alpha, \beta}+g_{i \beta, \alpha}-g_{\alpha \beta, i}\right)+\frac{1}{2} g^{\mu 0}\left(g_{0 \alpha, \beta}+g_{0 \beta, \alpha}-g_{\alpha \beta, 0}\right),
$$

we have the equation

$$
\frac{\mathrm{d} u^{\mu}}{\mathrm{d} \tau}+\Gamma_{\alpha \beta}^{\mu} u^{\alpha} u^{\beta}-g^{\mu 0} g_{0 \alpha} \frac{\mathrm{d} u^{\alpha}}{\mathrm{d} \tau}-\frac{1}{2} g^{\mu 0}\left(g_{0 \alpha, \beta}+g_{0 \beta, \alpha}-g_{\alpha \beta, 0}\right) u^{\alpha} u^{\beta}=0 .
$$

We claim that

$$
u_{\mu}\left(\frac{\mathrm{d} u^{\mu}}{\mathrm{d} \tau}+\Gamma_{\alpha \beta}^{\mu} u^{\alpha} u^{\beta}\right)=0
$$


To prove this identity, we replace $\Gamma_{\alpha \beta}^{\mu}$ by its defined value, multiply through by $u_{\mu}$ and then use the antisymmetry of the last two terms in the resulting expression to conclude that

$$
u_{\mu}\left(\frac{\mathrm{d} u^{\mu}}{\mathrm{d} \tau}+\Gamma_{\alpha \beta}^{\mu} u^{\alpha} u^{\beta}\right)=u_{\mu} \frac{\mathrm{d} u^{\mu}}{\mathrm{d} \tau}+\frac{1}{2} u^{\rho} g_{\rho \alpha, \beta} u^{\alpha} u^{\beta} .
$$

A calculation shows that the right-hand side of this last equation is equal to

$$
\frac{1}{2} \frac{\mathrm{d}}{\mathrm{d} \tau}\left(g_{\mu \nu} u^{\mu} u^{\nu}\right)
$$

and therefore it is equal to zero, since $(\mathrm{d} s / \mathrm{d} \tau)^{2}=g_{\mu \nu} u^{\mu} u^{\nu}=-1$.

After multiplying equation (2.7) by $u_{\mu}$, we use equation (2.8) to see that

$$
g_{0 \alpha} \frac{\mathrm{d} u^{\alpha}}{\mathrm{d} \tau}+\frac{1}{2}\left(g_{0 \alpha, \beta}+g_{0 \beta, \alpha}-g_{\alpha \beta, 0}\right) u^{\alpha} u^{\beta}=0,
$$

since $u_{\mu} g^{\mu 0}=u^{0}=\mathrm{d} t / \mathrm{d} \tau \neq 0$. By inserting this equality into equation (2.7), it follows that

$$
\frac{\mathrm{d} u^{\mu}}{\mathrm{d} \tau}+\Gamma_{\alpha \beta}^{\mu} u^{\alpha} u^{\beta}=0
$$

In particular, if $\mu=0$, then

$$
\frac{\mathrm{d}^{2} t}{\mathrm{~d} \tau^{2}}+\Gamma_{\alpha \beta}^{0} u^{\alpha} u^{\beta}=0
$$

and if $\mu=i$, then

$$
\frac{\mathrm{d}^{2} x^{i}}{\mathrm{~d} \tau^{2}}+\Gamma_{\alpha \beta}^{i} u^{\alpha} u^{\beta}=0
$$

Using the chain rule, we find that

$$
\frac{\mathrm{d}^{2} x^{i}}{\mathrm{~d} \tau^{2}}=\left(\frac{\mathrm{d} t}{\mathrm{~d} \tau}\right)^{2} \frac{\mathrm{d}^{2} x^{i}}{\mathrm{~d} t^{2}}+\frac{\mathrm{d}^{2} t}{\mathrm{~d} \tau^{2}} \frac{\mathrm{d} x^{i}}{\mathrm{~d} t} .
$$

By inserting this identity into equation (2.10) and by replacing $\mathrm{d} x^{\rho} / \mathrm{d} \tau$ with $\left(\mathrm{d} x^{\rho} / \mathrm{d} t\right)(\mathrm{d} t / \mathrm{d} \tau)$, we obtain equation (2.5) with its left-hand side multiplied by the nonzero factor $(\mathrm{d} t / \mathrm{d} \tau)^{2}$. This proves the desired result.

\subsection{Generalized Jacobi equation}

The generalized Jacobi equation is the linearization of the reduced geodesic equation (2.5) in Fermi coordinates with respect to the space variables, but not their velocities. We will obtain the explicit expression for this equation in Fermi coordinates valid in a cylindrical spacetime region along the worldline of a reference observer, which is at rest at the spatial origin of the Fermi coordinates (see appendix A). This is perhaps the most useful form of the generalized Jacobi equation.

For an arbitrary spacetime, when a reference observer (follows a geodesic) in a coordinate chart $\left(t, x^{i}\right)$ is specified, we can always find a Fermi coordinate system $\left(T, X^{i}\right)$ in a neighbourhood of the worldline of the fiducial observer so that the metric tensor is given by

$$
\begin{aligned}
& g_{00}=-1-{ }^{F} R_{0 i 0 j}(T) X^{i} X^{j}+\cdots, \\
& g_{0 i}=-\frac{2}{3}{ }^{F} R_{0 j i k}(T) X^{j} X^{k}+\cdots, \\
& g_{i j}=\delta_{i j}-\frac{1}{3}{ }^{F} R_{i k j \ell}(T) X^{k} X^{\ell}+\cdots,
\end{aligned}
$$

where

$$
{ }^{F} R_{\alpha \beta \gamma \delta}=R_{\mu \nu \rho \sigma} \lambda_{(\alpha)}^{\mu} \lambda_{(\beta)}^{\nu} \lambda_{(\gamma)}^{\rho} \lambda_{(\delta)}^{\sigma},
$$


are the components of the Riemann tensor projected onto the orthonormal tetrad frame $\lambda_{(\alpha)}^{\mu}(T)$ that is parallel propagated along the worldline of the reference observer at rest at the spatial origin of the Fermi chart (cf appendix A). By expanding the coefficients of the reduced geodesic equation (2.5) for the metric expressed in the Fermi coordinates and retaining only the first-order terms in the spatial directions, we obtain the generalized Jacobi equation

$$
\begin{aligned}
\frac{\mathrm{d}^{2} X^{i}}{\mathrm{~d} T^{2}}+{ }^{F} R_{0 i 0 j} & X^{j}+2^{F} R_{i k j 0} V^{k} X^{j} \\
& +\left(2^{F} R_{0 k j 0} V^{i} V^{k}+\frac{2}{3}{ }^{F} R_{i k j \ell} V^{k} V^{\ell}+\frac{2}{3}{ }^{F} R_{0 k j \ell} V^{i} V^{k} V^{\ell}\right) X^{j}=0,
\end{aligned}
$$

where $V^{i}:=\mathrm{d} X^{i} / \mathrm{d} T$. Alternatively, we could start from the Hamiltonian equations (2.4) and substitute in the Hamiltonian for $g^{00}, g^{0 i}$ and $\tilde{g}^{i j}$ expansions corresponding to equations (2.11) and recover equation (2.12) directly from Hamilton's equations (2.4); this is done in appendix B. The generalized Jacobi equation in arbitrary spacetime coordinates is given in appendix $\mathrm{C}$.

It is interesting to note a special scaling property of equation (2.12). To this end, we let $\varrho$ denote the radius of curvature; it is defined here so that $1 / \varrho^{2}$ is the supremum of the absolute magnitudes of the components of the Riemann tensor over the coordinate patch where the Fermi coordinates are defined. We will use the natural rescaling of the generalized Jacobi equation given by

$$
X^{i}=\varrho \hat{x}^{i}, \quad T=\varrho \hat{t} .
$$

In fact, under this rescaling the generalized Jacobi equation maintains its form except that each component of the Riemann tensor is multiplied by $\varrho^{2}$. The scaled equation is valid for $\left|\hat{x}^{i}\right| \ll 1$ and for arbitrary velocities whose magnitudes do not exceed the speed of light $c=1$. The requirement that $|\hat{\boldsymbol{x}}| \ll 1$ would naturally imply that in most cases the generalized Jacobi equation would hold for a limited interval of time $\hat{t}-\hat{t}_{0}$ starting from some initial state at $\hat{t}=\hat{t}_{0}$ such that $\left|\hat{t}-\hat{t}_{0}\right| \lesssim 1$.

The Hamiltonian dynamics of the generalized Jacobi equation will be explored in the following section for relative motion in the field of a plane gravitational wave. The dimensionless Fermi coordinates $(\hat{t}, \hat{\boldsymbol{x}})$ introduced in equation (2.13) will only be employed in the latter part of section 3 and should not be confused with the usual local coordinates that are used to express the spacetime metric of the plane wave below.

\section{Generalized Jacobi equation for a plane gravitational wave}

In this section, we will determine the generalized Jacobi equation for a plane gravitational wave given by the metric

$$
\mathrm{d} s^{2}=-\mathrm{d} t^{2}+\mathrm{d} x^{2}+U^{2}\left(\mathrm{e}^{2 h} \mathrm{~d} y^{2}+\mathrm{e}^{-2 h} \mathrm{~d} z^{2}\right),
$$

where $U$ and $h$ are functions of retarded time $u=t-x$. This metric is a solution of Einstein's field equations in vacuum $\left(R_{\mu \nu}=0\right)$ provided that

$$
U_{, u u}+\left(h_{, u}\right)^{2} U=0 .
$$

Under this assumption, the gravitational field is of Petrov type $N$ (see [6]) and represents a plane wave propagating along the positive $x$-axis.

When $|h| \ll 1$ and $U=1$, the metric reduces to a linear plane gravitational wave in the $T-T$ gauge that is linearly polarized (' $\oplus$ ').

Any observer that remains at rest at spatial coordinates $(x, y, z)$ follows a geodesic of this spacetime. This is a consequence of a more general result discussed in appendix D. 
Let us consider the reference observer at rest at the spatial origin. We claim that the orthonormal tetrad frame of this observer

$$
\begin{array}{ll}
\lambda_{(0)}^{\mu}=(1,0,0,0), & \lambda_{(1)}^{\mu}=(0,1,0,0), \\
\lambda_{(2)}^{\mu}=\left(0,0, U^{-1} \mathrm{e}^{-h}, 0\right), & \lambda_{(3)}^{\mu}=\left(0,0,0, U^{-1} \mathrm{e}^{h}\right)
\end{array}
$$

is parallel propagated along its worldline; that is,

$$
\frac{\mathrm{d} \lambda_{(\alpha)}^{\mu}}{\mathrm{d} t}+\Gamma_{\rho \sigma}^{\mu} \lambda_{(0)}^{\rho} \lambda_{(\alpha)}^{\sigma}=0 .
$$

It follows from appendix D that the worldline of this observer lies on a geodesic (that is, the last equation holds for $\alpha=0$ ), so it suffices to show that the equation holds for $\alpha=i$. In fact, because the nonzero components of $\Gamma_{0 \alpha}^{i}$ are

$$
\Gamma_{02}^{2}=\frac{1}{2} \frac{g_{22,0}}{g_{22}}, \quad \Gamma_{03}^{3}=\frac{1}{2} \frac{g_{33,0}}{g_{33}},
$$

it is easy to see that $\lambda_{(1)}^{\mu}$ satisfies the equation. The desired result for $\lambda_{(2)}^{\mu}$ and $\lambda_{(3)}^{\mu}$ follows from the fact that $U^{-1} \exp (-h)$ and $U^{-1} \exp (h)$ in equation (3.2) are respectively equal to $\left(g_{22}\right)^{-1 / 2}$ and $\left(g_{33}\right)^{-1 / 2}$ up to a sign factor.

To obtain the generalized Jacobi equation for the reference observer, we must compute the Fermi components of curvature along its worldline. Since the observer is at rest at the origin ( $x=y=z=0$ ), these components will be functions of its proper time $t=T$ only. In fact, for

$$
K(u):=h_{, u u}+2 h_{, u} \frac{U_{, u}}{U}
$$

we have that

$$
\begin{aligned}
& { }^{F} R_{1212}={ }^{F} R_{1220}={ }^{F} R_{2020}=-K(T), \\
& { }^{F} R_{1313}={ }^{F} R_{1330}={ }^{F} R_{3030}=K(T)
\end{aligned}
$$

are the only nonzero components except for the symmetries of the Riemann tensor. In the Fermi system, the spatial coordinates $\boldsymbol{X}$ are characterized invariantly with respect to the tetrad system as described in appendix A.

By inserting the curvature components into the generalized Jacobi equation (2.12) with $\dot{X}^{i}=\mathrm{d} X^{i} / \mathrm{d} T$, we have the following system of second-order differential equations:

$\ddot{X}=-\frac{2}{3} K(T)(\dot{X}-1)\left[X\left(\dot{Y}^{2}-\dot{Z}^{2}\right)-Y \dot{Y}(\dot{X}-3)+Z \dot{Z}(\dot{X}-3)\right]$,

$\ddot{Y}=-\frac{1}{3} K(T)\left\{2 X \dot{Y}\left(\dot{X}+\dot{Y}^{2}-\dot{Z}^{2}\right)-Y\left[3+2 \dot{Y}^{2}(\dot{X}-3)-6 \dot{X}+2 \dot{X}^{2}\right]+2 Z \dot{Y} \dot{Z}(\dot{X}-3)\right\}$,

$\ddot{Z}=\frac{1}{3} K(T)\left\{2 X \dot{Z}\left(\dot{X}-\dot{Y}^{2}+\dot{Z}^{2}\right)+2 Y \dot{Y} \dot{Z}(\dot{X}-3)-Z\left[3+2 \dot{Z}^{2}(\dot{X}-3)-6 \dot{X}+2 \dot{X}^{2}\right]\right\}$.

We note that this system is invariant under the transformation $X \mapsto X, Y \mapsto Z, Z \mapsto Y$ and $K \mapsto-K$. The generalized Jacobi system (3.3) with its velocity-dependent nonlinearities may conceivably have practical applications in the future for the case of space-based gravitational wave detectors such as LISA [7].

\subsection{Dynamics}

System (3.3) has a seven-dimensional extended state space such that the five-dimensional submanifolds

$$
\begin{aligned}
& M_{Y}:=\{(X, Y, Z, \dot{X}, \dot{Y}, \dot{Z}, T): Y=\dot{Y}=0\}, \\
& M_{Z}:=\{(X, Y, Z, \dot{X}, \dot{Y}, \dot{Z}, T): Z=\dot{Z}=0\}
\end{aligned}
$$


are invariant. The extended state space is simply the space of positions, velocities and time. On the three-dimensional invariant intersection of these manifolds, the flow is given by $\ddot{X}=0$ and $\dot{T}=1$. That is, equations (3.3) permit the uniform motion of a particle along the direction of wave propagation relative to the fiducial particle. This is due to the transverse character of the gravitational wave. Since the corresponding two-parameter family of solutions is $X(T)=\dot{X}(0) T+X(0)$, the relative motion is always unstable. That is, most members of this two-parameter family of geodesics diverge from the reference geodesic, which is the member of this family with $X(0)=\dot{X}(0)=0$.

In the small- $h$ approximation for a monochromatic wave with $U=1$, the curvature is given by $K=h_{, u u}$; hence, the assumption that $h(u)=-\epsilon \cos (\phi+\Omega u)$ results in the curvature $K(T)=\epsilon \Omega^{2} \cos (\phi+\Omega T)$, where $\epsilon \ll 1, \Omega$ is the wave frequency and $\phi$ is a constant phase. In this case, the radius of curvature is $\varrho=1 /(\Omega \sqrt{\epsilon})$ and, using the scaled variables (2.13), system (3.3) maintains its form except that $K(T)$ is replaced by $k(\hat{t}):=\cos (\phi+\hat{t} / \mu)$, where $\mu:=\sqrt{\epsilon}$. It is convenient, therefore, to define a new temporal variable $S:=\hat{t} / \mu$ and consider the equivalent first-order system with

$$
\hat{x}^{\prime}=\mu \xi, \quad \hat{y}^{\prime}=\mu \eta, \quad \hat{z}^{\prime}=\mu \zeta,
$$

where a prime denotes differentiation with respect to the new 'fast' time $S$. Each component of the vector field corresponding to the resulting first-order system has order $\mu$ and is $2 \pi$-periodic. This system is, therefore, in the correct form for averaging with respect to the temporal variable.

By averaging to second order in the small parameter $\mu$, we transform system (3.3) (by a near-identity nonautonomous change of variables) to a system that is autonomous to second order in $\mu$. In fact, the second-order approximation of the averaged system is given by

$$
\begin{aligned}
& \hat{x}^{\prime}=\mu \xi, \\
& \xi^{\prime}=0, \\
& \hat{y}^{\prime}=\mu \eta-\mu^{2} y \sin \phi, \\
& \eta^{\prime}=\mu^{2} \eta \sin \phi, \\
& \hat{z}^{\prime}=\mu \zeta+\mu^{2} z \sin \phi, \\
& \zeta^{\prime}=-\mu^{2} \zeta \sin \phi .
\end{aligned}
$$

This second-order approximation is not affected by the nonlinear terms in the generalized Jacobi equation; that is, the result would be the same for the corresponding Jacobi equation. The effect of the nonlinearities first appears in the third-order averaged system, i.e., at order $\epsilon^{3 / 2}$.

Let us note that taking into account the first-order approximation in $\mu$ only, the approximation procedure suggests that on average $X, Y$ and $Z$ are not affected by the presence of the wave; that is, to first order in $\mu, \boldsymbol{X}(T)=\boldsymbol{X}(0)+\dot{\boldsymbol{X}}(0) T$. In case $\sin \phi \neq 0$, the first correction to this approximation-necessary only for $Y(T)$ and $Z(T)$-is obtained from the second-order approximation of the averaged system (3.4); in fact, we find that

$$
\begin{aligned}
Y(T) & =Y(0) \mathrm{e}^{-\epsilon \Omega T \sin \phi}+\frac{\dot{Y}(0)}{\epsilon \Omega \sin \phi} \sinh (\epsilon \Omega T \sin \phi) \\
& =Y(0)+\dot{Y}(0) T-\epsilon Y(0) \Omega T \sin \phi+O\left(\epsilon^{2}\right), \\
Z(T) & =Z(0) \mathrm{e}^{\epsilon \Omega T \sin \phi}+\frac{\dot{Z}(0)}{\epsilon \Omega \sin \phi} \sinh (\epsilon \Omega T \sin \phi) \\
& =Z(0)+\dot{Z}(0) T+\epsilon Z(0) \Omega T \sin \phi+O\left(\epsilon^{2}\right) .
\end{aligned}
$$


It follows from these results that for $\sin \phi=0$, the motion is on average unaffected by the presence of the wave to first order in $\epsilon$, as expected from the form of equation (3.4). Moreover, equations (3.5) ignore nonlinearities in the system (3.3) and are therefore valid for the Jacobi equation, averaged to order $\epsilon$. It should be noted that for the Jacobi equation, the motions in $Y$ and $Z$ are stable as follows from an analysis of the corresponding Mathieu equations. This is not in conflict with equations (3.5) since to see this stability one has to average to higher orders than $\mu^{2}=\epsilon$.

\subsection{Hamiltonian chaos}

The dynamical behaviour of the generalized Jacobi system (3.3) is complicated by its high dimensionality and the velocity-dependent nonlinear terms. On the other hand, the system is Hamiltonian and if $K$ is periodic, we expect some dynamical effects associated with Hamiltonian chaos: resonance phenomena, stochastic regions and diffusion. While an investigation of these phenomena is beyond the scope of this paper, we illustrate the existence of Hamiltonian chaos in generalized Jacobi equations for the simpler system with $1+1 / 2$ degrees of freedom given by

$$
\frac{\mathrm{d}^{2} X}{\mathrm{~d} T^{2}}=K(T)\left(1-2 V^{2}\right) X
$$

where $V:=\mathrm{d} X / \mathrm{d} T$ and $K$ is a periodic function. This equation of relative motion is obtained from the generalized Jacobi equation by limiting attention to a two-dimensional world, i.e., a surface $(T, X)$, where $K$ is the Gaussian curvature of the surface (cf section 4$)$. According to the results of appendix B, equation (3.6) can be obtained from a Hamiltonian

$$
H(T, X, p)=\mp\left(1+p^{2}\right)^{1 / 2}\left[1+\frac{1}{2} K(T) X^{2}\right],
$$

once only terms linear in $X$ are retained in the equation of relative motion.

It is interesting to remark that the equivalent first-order system

$$
\dot{X}=V, \quad \dot{V}=K(T)\left(1-2 V^{2}\right) X,
$$

is Hamiltonian with respect to the symplectic form

$$
\omega=\left(1-2 V^{2}\right)^{-1 / 2} \mathrm{~d} X \wedge \mathrm{d} V,
$$

in the region of the state space where $V^{2} \neq 1 / 2$. Also, we note that the change of coordinates

$$
x=X, \quad v=\frac{1}{2 \sqrt{2}} \ln \left(\frac{1+\sqrt{2} V}{1-\sqrt{2} V}\right),
$$

for $V^{2} \neq 1 / 2$, transforms system (3.7) to

$$
\dot{x}=\frac{1}{\sqrt{2}}\left(\frac{\exp (2 \sqrt{2} v)-1}{\exp (2 \sqrt{2} v)+1}\right), \quad \dot{v}=-K(T) x,
$$

a system that is Hamiltonian with respect to the usual symplectic structure.

A few typical orbits with $|V|<(1 / 2)^{1 / 2}$ are illustrated in figure 1 for a stroboscopic Poincaré map obtained by numerical integration of system (3.7) with $K(T)=0.5 \cos (T)$; that is, we plot $V$ versus $X$ at times $2 n \pi$ for non-negative integer values of $n$. We note the presence of stochastic layers, resonant island chains and invariant closed curves. The proof of the existence of chaotic invariant sets even in this two-dimensional case is not simple since a straightforward application of the Melnikov method runs into standard difficulties; in particular, one has to deal with the problem of exponentially small splittings of homoclinic loops. 


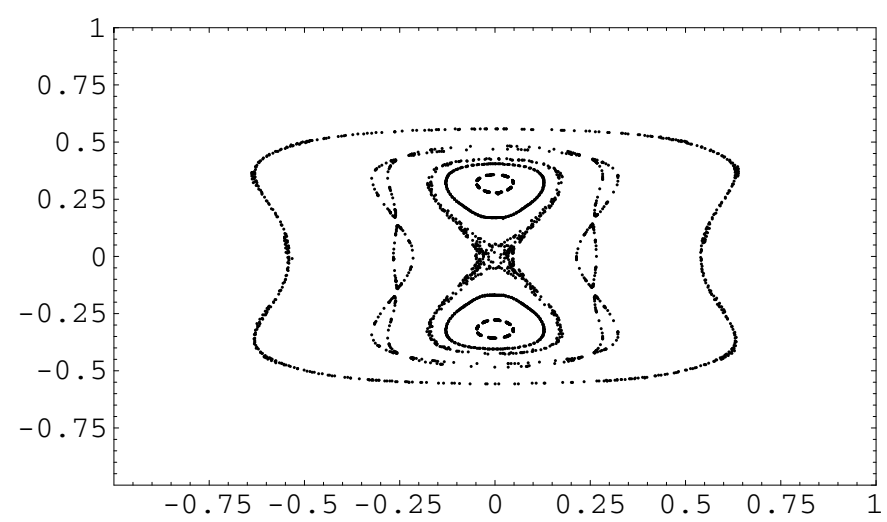

Figure 1. The plot of $V$ versus $X$ at $T=2 n \pi$ for $n=0,1,2, \ldots, 600$ is depicted for several orbits of the Poincaré map for system (3.6) with $K(T)=0.5 \cos (T)$.

\section{Generalized Jacobi equation for radial motion in black-hole spacetimes}

Imagine the relative motion of nearby free test particles along the axis of rotational symmetry of a Kerr source. For the reference geodesic, the equation of motion, using the standard Boyer-Lindquist radial coordinate $r$, is [2]

$$
\left(\frac{\mathrm{d} r}{\mathrm{~d} \tau}\right)^{2}=\gamma^{2}-1+\frac{2 G M r}{r^{2}+a^{2}},
$$

where $G$ is Newton's gravitational constant, $M$ is the mass of the Kerr source and $a M$ is its angular momentum. Here $\gamma$ is a positive constant of integration and $\gamma>1$ has the interpretation of the Lorentz factor for the particle at infinity $(r \rightarrow \infty)$.

For a neighbouring geodesic, the generalized Jacobi equation describing motion along the axis relative to the fiducial observer is

$$
\frac{\mathrm{d}^{2} R}{\mathrm{~d} T^{2}}+k(T)\left[1-2\left(\frac{\mathrm{d} R}{\mathrm{~d} T}\right)^{2}\right] R=0
$$

where $\boldsymbol{X}=(R, 0,0), R$ is the relative radial distance along the axis, $T=\tau$ along the reference geodesic and $k(T)$ is obtained [2] from the solution of equation (4.1) and

$$
k=-2 \frac{G M r\left(r^{2}-3 a^{2}\right)}{\left(r^{2}+a^{2}\right)^{3}} .
$$

It is interesting to note that in this form $k$ is independent of boosts along the axis. This is a consequence of the fact that the Kerr solution is of Petrov type D and hence the Kerr symmetry axis provides two special tidal directions for ingoing and outgoing trajectories [3, 8]. For $a=0$, the problem reduces to purely radial relative motion in the exterior Schwarzschild spacetime.

More generally, starting from the generalized Jacobi equation and limiting our attention to a two-dimensional world as in section 3.2, let us consider the equation of relative motion in the form

$$
\frac{\mathrm{d}^{2} X}{\mathrm{~d} T^{2}}+\kappa\left(1-2 V^{2}\right) X=0,
$$

where $\kappa(T), \kappa={ }^{F} R_{0101}$, is the Gaussian curvature of the surface $(X, T)$ and $V=\mathrm{d} X / \mathrm{d} T$. 


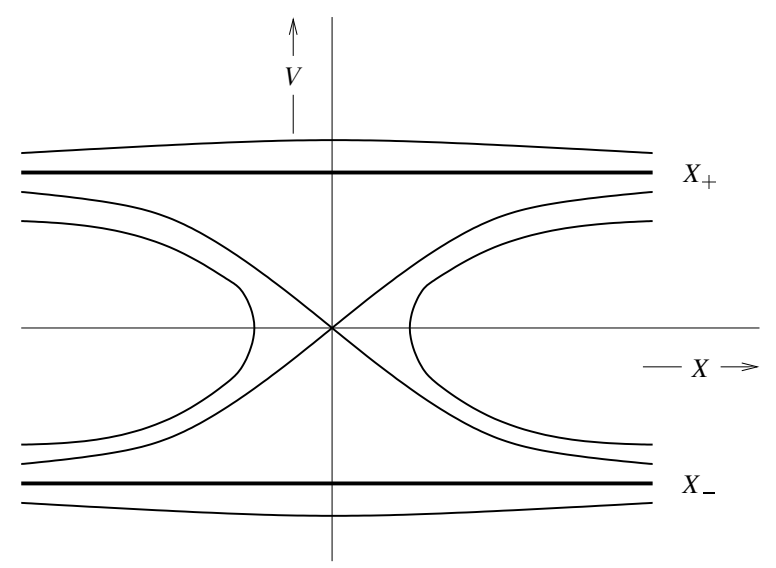

Figure 2. Phase diagram of equation (4.4) for negative constant curvature. The bold horizontal lines correspond to $X_{ \pm}$.

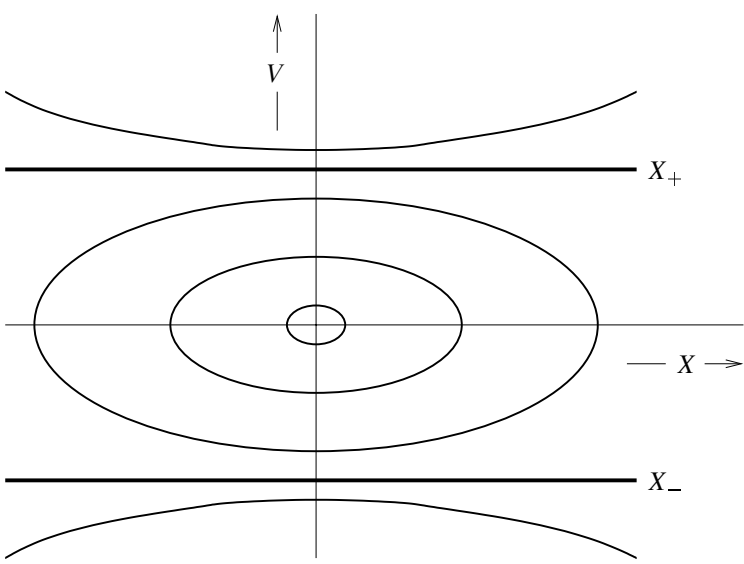

Figure 3. Phase diagram of equation (4.4) for positive constant curvature. The bold horizontal lines correspond to $X_{ \pm}$.

To investigate the main consequences of equation (4.4) beyond the original Jacobi equation for arbitrary curvature, we assume that $|V|,-1<V<1$, is not negligibly small compared to unity. In particular, let us note that equation (4.4) has constant velocity solutions with $V^{2}=1 / 2$ regardless of the curvature of the surface, i.e., $X_{ \pm}=X_{0} \pm(1 / 2)^{1 / 2}\left(T-T_{0}\right)$, where $T_{0}$ is a constant initial time and $X_{0}=X\left(T_{0}\right)$. It is, therefore, interesting to investigate the general behaviour of solutions of equation (4.4) near these constant velocity solutions. The asymptotic behaviour of nearby solutions is determined by the curvature. Figures 2 and 3 depict the phase portraits of system (4.4) for constant curvature cases $\kappa<0$ and $\kappa>0$, respectively. We note that for constant $\kappa$, equation (4.4) is completely integrable. It would be interesting to investigate the behaviour of the solutions of equation (4.4) near $X_{ \pm}$for arbitrary $\kappa(T)$. In the rest of this section, we limit our attention to $\kappa(T)<0$, since it follows from equation (4.3) that this is the relevant case for motion away from a Kerr source $\left(r^{2}>3 a^{2}\right)$.

For the negative curvature case, we consider the asymptotic stability of the invariant sets $\mathcal{X}_{ \pm}:=\left\{(X, V, T): V= \pm(1 / 2)^{1 / 2}\right\}$ in the extended state space for equation (4.4). 
For example, we call $\mathcal{X}_{+}$asymptotically stable if for every solution $(X(T), V(T), T)$ in the extended state space of equation (4.4) that starts near $\mathcal{X}_{+}$, its distance to $\mathcal{X}_{+}$along the velocity direction decreases to zero as $T$ increases to infinity. It can be shown that asymptotic stability is essentially determined by the linearized first-order system along $\mathcal{X}_{+}$, i.e., the system involving the linear approximation $W$ of $X-X_{+}$and $Q$ of $V-(1 / 2)^{1 / 2}$ that is given by

$$
\frac{\mathrm{d} W}{\mathrm{~d} T}=Q, \quad \frac{\mathrm{d} Q}{\mathrm{~d} T}=2 \kappa(T)\left[\sqrt{2} X_{0}+\left(T-T_{0}\right)\right] Q .
$$

Integrating system (4.5), we find that the invariant set $\mathcal{X}_{+}$of equation (4.4) is positively asymptotically stable if for each real number $C_{0}$ we have

$$
\int_{T_{0}}^{\infty} \kappa(T)\left(T+C_{0}\right) \mathrm{d} T=-\infty
$$

This result has a useful corollary: if $\kappa(T)$ is asymptotic to a negative constant multiple of $T^{-p}$ with $p \leqslant 2$ as $T \rightarrow \infty$, then $\mathcal{X}_{+}$is asymptotically stable.

Let us note that if $\gamma<1$ in the differential equation (4.1), then there is a real solution only if the right-hand side of the differential equation is positive. In this case the real solutions with $r>0$ remain bounded between the two positive roots of the right-hand side. Under the additional assumption that the square of the smaller positive root exceeds $3 a^{2}$, the curvature obtained from equation (4.3) is bounded below zero; therefore, the integral condition (4.6) is satisfied and $\mathcal{X}_{+}$is positively asymptotically stable. For $\gamma=1$, by using an asymptotic analysis of equation (4.1) we find that $r(T)$ is asymptotic to a constant multiple of $T^{2 / 3}$ as $T \rightarrow \infty$ and $\kappa(T)$ is, therefore, asymptotic to a negative constant multiple of $T^{-2}$. Thus, for $\gamma=1$ the invariant set $\mathcal{X}_{+}$is again asymptotically stable; the rate of attraction, however, is slower than any exponential rate. For $\gamma>1$, the set $\mathcal{X}_{+}$is no longer asymptotically stable. Although nearby solutions in this case move closer to $\mathcal{X}_{+}$as $T \rightarrow \infty$, they are bounded away from this manifold. Let us note that the results of this analysis also apply to the behaviour of solutions near $\mathcal{X}_{-}$, since in this case the linearized system is identical to equation (4.5) except for $X_{0} \mapsto-X_{0}$.

The $\kappa<0$ case is particularly interesting in connection with the problem of 'superluminal' jets in astrophysics [9]. In fact, the Galactic 'superluminal' jet sources GRS 1915+105 and GRO J1655-40 have speeds that may be near $(1 / 2)^{1 / 2} \approx 0.7$.

\section{Discussion}

The main dynamical features of the generalized Jacobi equation have been presented in this paper. In contrast to the standard Jacobi equation, the generalized Jacobi equation is nonlinear and therefore exhibits Hamiltonian chaos under certain circumstances. Two specific applications have been considered: relative test particle motion in the field of a plane gravitational wave and in the field of a rotating mass. The results may be relevant respectively to future space-based gravitational wave detectors and high-energy astrophysical phenomena associated with jets.

The bulk speed of Galactic 'superluminal' jets appears to be constant and estimated to be around $90 \%$ of the speed of light, though many uncertainties are associated with this estimate [9]. Jet motion is detected via the temporal evolution of the radiation intensity contours relative to the background, which would indicate the motion of blobs (electron clouds) in the jet relative to the ambient medium. This motion, under the influence of gravitation alone, is described by the generalized Jacobi equation as discussed in detail in the previous section. In this case, the generalized Jacobi equation exhibits an attractor with constant speed $2^{-1 / 2}$, approximately equal to $70 \%$ of the speed of light. 
Assuming that far from the source gravitational tidal forces are dominant, it follows from our results that the jet speed should approach $2^{-1 / 2}$ from above and below over a certain characteristic timescale. This timescale can be calculated from the solution of equation (4.5) for $Q$. More explicitly, let us consider the jet along the axis of a Kerr black hole of mass $M$ and angular momentum $J \leqslant M^{2}$ at a distance $r_{0} \gg 2 G M$; then, the characteristic timescale is $\rho_{0}$, where $\rho_{0}$ is the radius of curvature at $r_{0}$ given by $\rho_{0} \approx\left(r_{0}^{3} / 2 G M\right)^{1 / 2}$. Simple estimates suggest that further refinements of the observational techniques [9] are required before it may be possible to test this prediction of the elementary theory of the asymptotic jet speed presented in this paper.

\section{Appendix A. Fermi coordinate systems}

Consider a reference observer following a curve $C$ in spacetime. Let $\tau$ be the proper time along $C$ and $u^{\mu}=\mathrm{d} x^{\mu} / \mathrm{d} \tau$ be its tangent vector. The fiducial observer carries a triad $\lambda_{(i)}^{\mu}$ representing three ideal gyroscope directions so that $\lambda_{(i)}^{\mu}$ are parallel transported and $\lambda_{(\alpha)}^{\mu}$ with $\lambda_{(0)}^{\mu}=u^{\mu}$ is an orthonormal tetrad frame. The Fermi system is a geodesic coordinate system valid in a cylindrical spacetime region around $C$ such that for any point $P$ with Fermi coordinates $X^{\alpha}=\left(T, X^{i}\right)$, there exists a unique spacelike geodesic orthogonal to $C$ that connects $P$ with a point $P_{0}$ on $C$. Let $\tau$ be the proper time at $P_{0}, \sigma$ be the proper length of the spacelike geodesic starting from $P_{0}$ and $\eta^{\mu}=\left(\mathrm{d} x^{\mu} / \mathrm{d} \sigma\right)_{P_{0}}$ be the vector tangent to the spacelike geodesic at $P_{0}$; then, $\eta_{\mu} u^{\mu}=0$ and the Fermi coordinates are defined by

$$
T=\tau, \quad X^{i}=\delta^{i j} \sigma \eta_{\mu} \lambda_{(j)}^{\mu}
$$

(cf figure A1).

To determine the metric tensor $g_{\mu \nu}(x)$ in Fermi coordinates, i.e., $F_{g_{\mu \nu}}(X)$, we expand $F_{g_{\mu \nu}}(P)$ in a Taylor series about $F_{g_{\mu \nu}}\left(P_{0}\right)=\eta_{\mu \nu}$. In this expansion, the required derivatives of $F_{g_{\mu \nu}}$ at $P_{0}:(T, \mathbf{0})$ are calculated on the basis of the following considerations. The equations

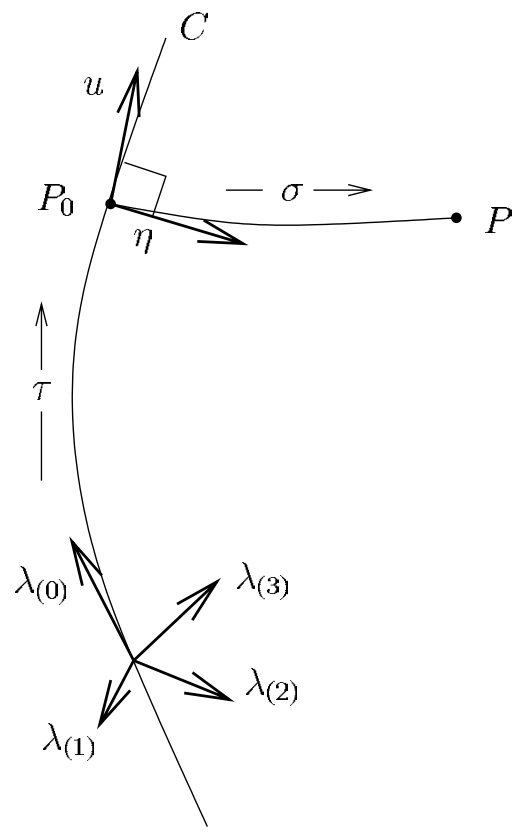

Figure A1. Schematic diagram depicting the construction of the Fermi normal coordinate system. 
of motion of the tetrad frame carried by the observer along the fiducial geodesic $C$ in Fermi coordinates reduce to ${ }^{F} \Gamma_{00}^{\mu}(T, \mathbf{0})=0$ and ${ }^{F} \Gamma_{0 i}^{\mu}(T, \mathbf{0})=0$. Moreover, the spacelike curve connecting $P_{0}$ to $P$ satisfies the geodesic equation, which in Fermi coordinates (A.1) reduces to ${ }^{F} \Gamma_{i j}^{\mu}(T, \mathbf{X}) X^{i} X^{j}=0$. Expanding ${ }^{F} \Gamma_{i j}^{\mu}$ in a Taylor series about $P_{0}:(T, \mathbf{0})$, we find that

$$
{ }^{F} \Gamma_{i j}^{\mu}(T, \mathbf{0})=0, \quad{ }^{F} \Gamma_{(i j, k)}^{\mu}(T, \mathbf{0})=0, \quad{ }^{F} \Gamma_{(i j, k \ell)}^{\mu}(T, \mathbf{0})=0, \ldots
$$

Using all these relations regarding the connection coefficients at $P_{0}$, the required derivatives of the metric tensor at $P_{0}$ can be calculated. The results can be expressed as

$$
\begin{aligned}
& F_{g_{00}}=-1-{ }^{F} R_{i 0 j 0} X^{i} X^{j}-\frac{2}{3}{ }^{F} \mathcal{R}_{i 0 j 0 k} X^{i} X^{j} X^{k}+\cdots, \\
& { }_{g_{0 i}}=-\frac{2}{3}{ }^{F} R_{0 j i k} X^{j} X^{k}+\frac{1}{2}{ }^{F} \mathcal{R}_{0 j k i \ell} X^{j} X^{k} X^{\ell}+\cdots, \\
& F_{g_{i j}}=\delta_{i j}-\frac{1}{3}{ }^{F} R_{i k j \ell} X^{k} X^{\ell}+\frac{1}{3}{ }^{F} \mathcal{R}_{i \ell r j k} X^{k} X^{\ell} X^{r}+\cdots,
\end{aligned}
$$

where $\mathcal{R}_{\mu \nu \rho \sigma \omega}$ is given in terms of covariant derivatives of the Riemann tensor by

$$
\mathcal{R}_{\mu \nu \rho \sigma \omega}=\frac{1}{2}\left(R_{\mu \nu \rho \sigma ; \omega}+R_{\mu \rho \omega \nu ; \sigma}\right) .
$$

It is simple to show that the tensor $\mathcal{R}_{\mu \nu \rho \sigma \omega}$ satisfies the following relations

$$
\mathcal{R}_{\mu \nu \rho \sigma \omega}=-\mathcal{R}_{\mu \rho \nu \omega \sigma}, \quad \mathcal{R}_{\mu \nu \rho \sigma \omega}-\mathcal{R}_{\mu \rho \omega \nu \sigma}=R_{\rho \sigma \mu(\nu ; \omega)} .
$$

The first covariant derivatives of the Riemann tensor are not completely specified by the tensor $\mathcal{R}_{\mu \nu \rho \sigma \omega}$. Moreover, the next terms in equations (A.3)-(A.5) contain, among other terms, the tensor

$$
\mathcal{T}_{\mu \nu \rho \sigma \omega \pi}=\frac{1}{3} \mathcal{R}_{\mu \nu \rho \sigma \omega ; \pi}+\frac{2}{3} R_{\rho \omega \nu}^{\xi} R_{\mu \xi \pi \sigma},
$$

etc. It follows from these results that the reduced geodesic equation (2.5) in Fermi coordinates becomes the tidal equation

$$
\frac{\mathrm{d}^{2} X^{i}}{\mathrm{~d} T^{2}}+K_{j}^{i} X^{j}+\frac{1}{2 !} K_{j k}^{i} X^{j} X^{k}+\frac{1}{3 !} K_{j k \ell}^{i} X^{j} X^{k} X^{\ell}+\cdots=0,
$$

where the quantities $K_{i j}, K_{i j k}$, etc, characterize, respectively, the tidal acceleration terms of first order, second order, etc. We find from equations (A.3)-(A.5) that

$$
K_{i j}={ }^{F} R_{0 i 0 j}-2{ }^{F} R_{0 j i k} \dot{X}^{k}-2\left({ }^{F} R_{0 j 0 k} \dot{X}^{i}-\frac{1}{3}{ }^{F} R_{i \ell j k} \dot{X}^{\ell}\right) \dot{X}^{k}-\frac{2}{3}{ }^{F} R_{0 \ell k j} \dot{X}^{i} \dot{X}^{\ell} \dot{X}^{k},
$$

as in the generalized Jacobi equation and $K_{i j k}=2 K_{i(j k)}^{\prime}$, where

$$
\begin{aligned}
K_{i j k}^{\prime}={ }^{F} \mathcal{R}_{i 0 j 0 k} & +2\left[{ }^{F} \mathcal{R}_{0 j \ell i k}-\frac{1}{3}\left({ }^{F} \mathcal{R}_{i j \ell k 0}+{ }^{F} \mathcal{R}_{0 i j k \ell}\right)\right] \dot{X}^{\ell}-{ }^{F} \mathcal{R}_{0 j 0 k 0} \dot{X}^{i} \\
& +\left({ }^{F} \mathcal{R}_{j \ell i k r}+\frac{5}{6}{ }^{F} \mathcal{R}_{k r i \ell j}\right) \dot{X}^{\ell} \dot{X}^{r}+\frac{2}{3}\left(2{ }^{F} \mathcal{R}_{0 j \ell 0 k}-{ }^{F} \mathcal{R}_{j 0 k 0 \ell}\right) \dot{X}^{i} \dot{X}^{\ell} \\
& +\frac{1}{6}\left({ }^{F} \mathcal{R}_{k \ell 0 j r}-5{ }^{F} \mathcal{R}_{\ell k 0 r j}\right) \dot{X}^{i} \dot{X}^{\ell} \dot{X}^{r} .
\end{aligned}
$$

The Jacobi equation in spaces of arbitrary dimensions was first discussed by Levi-Civita [10] and Synge [1, 11, 12]. These references contain a detailed and explicit exposition of the theorem of Fermi [13] involving the possibility of choosing a system of coordinates in a cylindrical region along an arbitrary open curve in spacetime such that all the connection coefficients vanish and $g_{\mu \nu}=\eta_{\mu \nu}$ on the curve.

The generalized Jacobi equation was first discussed in [14-16] and developed further in [17]. Later, this generalization was independently rediscovered by Ciufolini $[18,19]$. Further discussions of the Jacobi equation can be found, e.g., in [4, 20-22]. 


\section{Appendix B. Hamiltonian equations of motion}

Consider a coordinate system $X^{\alpha}=\left(T, X^{i}\right)$ in the region of interest in spacetime such that the metric tensor takes the form $g_{\mu \nu}=\eta_{\mu \nu}+h_{\mu \nu}$, where $h_{\mu \nu}$ is a small perturbation on the Minkowski background. In particular, we are interested in Fermi normal coordinates (2.11). The isoenergetically reduced Hamiltonian system is given in these coordinates by

$$
\frac{\mathrm{d} X^{i}}{\mathrm{~d} T}=\frac{\partial H}{\partial p_{i}}, \quad \frac{\mathrm{d} p_{i}}{\mathrm{~d} T}=-\frac{\partial H}{\partial X^{i}},
$$

where

$$
H=\frac{g^{0 i}}{g^{00}} p_{i} \mp\left(\frac{1+\tilde{g}^{i j} p_{i} p_{j}}{-g^{00}}\right)^{1 / 2} .
$$

To first order in $h_{\mu \nu}$, the Hamiltonian can be expressed as

$$
H=\mp \sqrt{1+p^{2}}\left(1-\frac{1}{2} h_{00}-\frac{1}{2} \frac{h^{i j} p_{i} p_{j}}{1+p^{2}}\right)+h^{0 i} p_{i}
$$

where $p^{2}=\delta^{i j} p_{i} p_{j}$ and $H$ is a quadratic form in spatial Fermi coordinates $\boldsymbol{X}$. Using (B.3), the system of equations (B.1) can be written as

$$
\begin{aligned}
& \frac{\mathrm{d} X^{i}}{\mathrm{~d} T}=\mp \frac{p_{j}}{\sqrt{1+p^{2}}}\left[\left(1-\frac{1}{2} h_{00}+\frac{1}{2} \frac{h^{k \ell} p_{k} p_{\ell}}{1+p^{2}}\right) \delta^{i j}-h^{i j}\right]+h^{0 i}, \\
& \frac{\mathrm{d} p_{i}}{\mathrm{~d} T}=\mp \frac{1}{2} \sqrt{1+p^{2}}\left(h_{00, i}+\frac{h^{k \ell}{ }_{i} p_{k} p_{\ell}}{1+p^{2}}\right)-h^{0 j}{ }_{, i} p_{j} .
\end{aligned}
$$

Letting $\mp p_{i} \rightarrow \hat{p}_{i}$ and defining

$$
P_{i}:=\frac{\hat{p}_{i}}{\sqrt{1+\hat{p}^{2}}}
$$

and $P^{i}:=\delta^{i j} P_{j}$, we find that $\left(1+\hat{p}^{2}\right)\left(1-P^{2}\right)=1$ and $\hat{p}_{i}=P_{i} / \sqrt{1-P^{2}}$. In terms of $\boldsymbol{P}$, the Hamiltonian equations of motion (B.4)-(B.5) take the form

$$
\begin{aligned}
& \frac{\mathrm{d} X^{i}}{\mathrm{~d} T}=P^{i}\left(1-\frac{1}{2} h_{00}+\frac{1}{2} h^{k \ell} P_{k} P_{\ell}\right)+h^{0 i}-h^{i j} P_{j}+\frac{1}{2} h^{k \ell} P_{k} P_{\ell} P^{i}, \\
& \sqrt{1-P^{2}} \frac{\mathrm{d}}{\mathrm{d} T}\left(\frac{P_{i}}{\sqrt{1-P^{2}}}\right)=\frac{1}{2} h_{00, i}-h^{0 j}{ }_{, i} P_{j}+\frac{1}{2} h^{k \ell}{ }_{, i} P_{k} P_{\ell} .
\end{aligned}
$$

We are interested in the behaviour of $X^{i}=X^{i}(T)$ to first order in $\delta=|\boldsymbol{X}| / \varrho$. To this end, we note from equation (B.7) that $P^{i}=V^{i}+O\left(h_{\mu \nu}\right)$, where $V^{i}=\mathrm{d} X^{i} / \mathrm{d} T$. Thus equation (B.7) can be written to first order in $h_{\mu \nu}$ as

$$
P_{i}:=\left(1+\frac{1}{2} h_{00}-\frac{1}{2} h_{k \ell} V^{k} V^{\ell}\right) V_{i}+h_{0 i}+h_{i j} V^{j} .
$$

Similarly, equation (B.8) can be written as

$$
\frac{\mathrm{d} P^{i}}{\mathrm{~d} T}+\frac{1}{1-P^{2}}\left(\boldsymbol{P} \cdot \frac{\mathrm{d} \boldsymbol{P}}{\mathrm{d} T}\right) P^{i}=\frac{1}{2} h_{00, i}+h_{0 j, i} V^{j}+\frac{1}{2} h_{k \ell, i} V^{k} V^{\ell} .
$$

This relation, after multiplying by $P_{i}$ and summing over $i$, takes the form

$$
\frac{1}{1-P^{2}}\left(\boldsymbol{P} \cdot \frac{\mathrm{d} \boldsymbol{P}}{\mathrm{d} T}\right)=\frac{1}{2} h_{00, i} V^{i}+h_{0 j, i} V^{i} V^{j}+\frac{1}{2} h_{k \ell, i} V^{k} V^{\ell} V^{i} .
$$


Substituting equations (B.11) and (B.9) in equation (B.10) and noting that

$$
\mathrm{d} V^{i} / \mathrm{d} T=O(\delta), \quad h_{\mu v, 0}=O\left(\delta^{2}\right),
$$

we finally obtain the desired equation of motion

$$
\begin{aligned}
\frac{\mathrm{d} V^{i}}{\mathrm{~d} T}+\left(h_{00, j} V^{j}\right. & \left.+h_{0 j, k} V^{j} V^{k}\right) V^{i}-\frac{1}{2} h_{00, i} \\
& +\left(h_{0 i, j}-h_{0 j, i}\right) V^{j}+\left(h_{i j, k}-\frac{1}{2} h_{j k, i}\right) V^{j} V^{k}=0 .
\end{aligned}
$$

Taking due account of the approximations under consideration here, we note that

$$
-\Gamma_{\alpha \beta}^{0} \frac{\mathrm{d} X^{\alpha}}{\mathrm{d} T} \frac{\mathrm{d} X^{\beta}}{\mathrm{d} T}=h_{00, j} V^{j}+h_{0 j, k} V^{j} V^{k}
$$

and

$$
\begin{aligned}
\Gamma_{\alpha \beta}^{i} \frac{\mathrm{d} X^{\alpha}}{\mathrm{d} T} \frac{\mathrm{d} X^{\beta}}{\mathrm{d} T} & =\Gamma_{00}^{i}+2 \Gamma_{0 j}^{i} V^{j}+\Gamma_{j k}^{i} V^{j} V^{k} \\
& =-\frac{1}{2} h_{00, i}+\left(h_{0 i, j}-h_{0 j, i}\right) V^{j}+\left(h_{i j, k}-\frac{1}{2} h_{j k, i}\right) V^{j} V^{k}
\end{aligned}
$$

in agreement with equation (2.5). Moreover, to first order in $\delta$

$$
\begin{aligned}
& h_{00, i}=-2 R_{0 i 0 j} X^{j}, \\
& h_{0 i, j}=-\frac{2}{3}\left(R_{0 j i k}+R_{0 k i j}\right) X^{k}, \\
& h_{\ell m, i}=-\frac{1}{3}\left(R_{\ell i m k}+R_{m i \ell k}\right) X^{k},
\end{aligned}
$$

in the Fermi system. Substituting these relations in equation (B.12) and using the symmetries of the Riemann tensor, such as $R_{0[i j k]}=0$, we obtain equation (2.12). This is the generalized Jacobi equation in Fermi coordinates; for arbitrary coordinates see appendix C.

\section{Appendix C. Generalized Jacobi equation in arbitrary coordinates}

Consider a reference timelike geodesic $C$ as in appendix A representing the worldline of an observer with proper time $\tau$. Let $\xi^{\mu}=\sigma \eta^{\mu}$; then, to first order in $\sigma / \varrho$, the generalized Jacobi equation $[14,15]$ takes the form

$$
\begin{gathered}
\frac{\mathrm{D}^{2} \xi^{\mu}}{\mathrm{D} \tau^{2}}+R_{\rho \nu \sigma}^{\mu} \\
u^{\rho} \xi^{\nu} u^{\sigma}+\left(u^{\mu}+\frac{\mathrm{D} \xi^{\mu}}{\mathrm{D} \tau}\right)\left(2 R_{\zeta \rho \nu \sigma} u^{\zeta} \frac{\mathrm{D} \xi^{\rho}}{\mathrm{D} \tau} \xi^{\nu} u^{\sigma}+\frac{2}{3} R_{\zeta \rho \nu \sigma} u^{\zeta} \frac{\mathrm{D} \xi^{\rho}}{\mathrm{D} \tau} \xi^{\nu} \frac{\mathrm{D} \xi^{\sigma}}{\mathrm{D} \tau}\right) \\
+2 R_{\rho \nu \sigma}^{\mu} \frac{\mathrm{D} \xi^{\rho}}{\mathrm{D} \tau} \xi^{\nu} u^{\sigma}+\frac{2}{3} R_{\rho \nu \sigma}^{\mu} \frac{\mathrm{D} \xi^{\rho}}{\mathrm{D} \tau} \xi^{\nu} \frac{\mathrm{D} \xi^{\sigma}}{\mathrm{D} \tau}=0 .
\end{gathered}
$$

Here

$$
\frac{\mathrm{D} \xi^{\mu}}{\mathrm{D} \tau}=\xi_{; \nu}^{\mu} \frac{\mathrm{d} x^{\nu}}{\mathrm{d} \tau}=\xi_{; \nu}^{\mu} u^{\nu}
$$

is the covariant derivative of $\xi^{\mu}$ along the reference worldline. If we now use Fermi coordinates and set

$$
\xi^{\mu}=X^{i} \lambda_{(i)}^{\mu}, \quad \frac{\mathrm{D} \xi^{\mu}}{\mathrm{D} \tau}=\frac{\mathrm{d} X^{i}}{\mathrm{~d} T} \lambda_{(i)}^{\mu}, \quad \frac{\mathrm{D}^{2} \xi^{\mu}}{\mathrm{D} \tau^{2}}=\frac{\mathrm{d}^{2} X^{i}}{\mathrm{~d} T^{2}} \lambda_{(i)}^{\mu}, \ldots,
$$

we recover equation (2.12). Here we have used the relation $\mathrm{D} \lambda_{(\alpha)}^{\mu} / \mathrm{D} \tau=0$; i.e., the tetrad is parallel transported along $C$. 


\section{Appendix D. Worldlines of observers at rest}

Consider a spacetime metric of the form

$$
\mathrm{d} s^{2}=-\mathrm{d} t^{2}+g_{i j}\left(t, x^{k}\right) \mathrm{d} x^{i} \mathrm{~d} x^{j} .
$$

If a particle is a rest at a point in the corresponding space, then its worldline follows a geodesic. Indeed, the 4-velocity of the particle $u^{\mu}=\mathrm{d} x^{\mu} / \mathrm{d} \tau$ is given by $u^{0}=1$ and $u^{i}=0$. We must show that

$$
\frac{\mathrm{d} u^{\mu}}{\mathrm{d} \tau}+\Gamma_{\alpha \beta}^{\mu} u^{\alpha} u^{\beta}=0
$$

For $\mu=0$, it suffices to prove that $\Gamma_{00}^{0}=0$. Because of the block form of the metric, we have $g^{00}=-1$ and $g^{0 i}=0$; therefore,

$$
\Gamma_{00}^{0}=-\frac{1}{2} g_{00,0}=0 .
$$

For $\mu=i$, it suffices to show that $\Gamma_{00}^{i}=0$. In this case we have $g_{0 j}=0$ and $g_{00}=-1$. Hence,

$$
\Gamma_{00}^{i}=\frac{1}{2} g^{i j}\left(2 g_{j 0,0}-g_{00, j}\right)=0,
$$

as required.

\section{References}

[1] Synge J L 1960 Relativity: the General Theory (Amsterdam: North-Holland)

[2] Mashhoon B 1982 Proc. 2nd Marcel Grossmann Meeting on General Relativity ed R Ruffini (New York: North-Holland) pp 647-72, see, in particular, section 4

[3] Beem J K, Ehrlich P E and Easley K L 1996 Global Lorentzian Geometry 2nd edn (New York: Dekker) ch 2

[4] Kerner R, van Holten J W and Colistete R Jr 2001 Class. Quantum Grav. 184725

[5] Abraham R and Marsden J 1985 Foundations of Mechanics 2nd edn (Reading, MA: Perseus) pp 223-5

[6] Bondi H, Pirani F A E and Robinson I 1959 Proc. R. Soc. A 251519

[7] The LISA mission is described in http://lisa.jpl.nasa.gov/ and http://www.estec.esa.nl/spdwww/future/html/lisa.htm

[8] Mashhoon B and McClune J C 1993 Mon. Not. R. Astron. Soc. 262881

[9] Fender R 2001 Preprint astro-ph/0109502

[10] Levi-Civita T 1926 Math. Ann. 97291

[11] Synge J L 1926 Phil. Trans. R. Soc. A 22631

[12] Synge J L 1935 Duke Math. J. 1527

[13] Fermi E 1992 Atti Accad. Naz. Lincei Rend. Cl. Sci. Fis. Mat. Nat. 31 21, 51

[14] Hodgkinson D E 1972 Gen. Rel. Grav. 3351

[15] Mashhoon B 1975 Astrophys. J. 197705

[16] Mashhoon B 1977 Astrophys. J. 216591

[17] Li W-Q and Ni W-T 1979 J. Math. Phys. 20 1473, 1925

[18] Ciufolini I 1986 Phys. Rev. D 341014

[19] Ciufolini I and Demiański M 1986 Phys. Rev. D 341018

[20] Bazański S L 1977 Ann. Inst. H. Poincaré 27 115, 145

[21] Alexandrov A N and Piragas K A 1979 Theor. Math. Phys. 3848

[22] Manoff S 2001 Int. J. Mod. Phys. A 161109 